

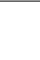




\section{Sistemas dinámicos en la interacción emocional madre-hijo: primera fase ${ }^{1}$}

Fecha de recepción: 7 de febrero de 2011

Jaime Castro Martínez

jcastrom@poli.edu.co

Politécnico Grancolombiano

Psicólogo de la Universidad Nacional de

Colombia. Docente y director del Laboratorio de

Psicología del Politécnico Grancolombiano.
Fecha de aceptación: 22 de agosto de 2011

\section{Resumen}

Los avances en psicología de las emociones y psicología del desarrollo han exigido nuevos cambios tanto de orden teórico como metodológico para describir y explicar la variabilidad de las res-puestas infantiles a los cambios que tienen lugar en la dinámica socio-emocional con la madre. Producto de estas variaciones, aparece la integración de los sistemas dinámicos a las descripciones de los teóricos del desarrollo emocional bajo

- supuestos psicobiológicos y sociobiológicos. Esta investigación indaga el posible uso de modelos de sistemas dinámicos en la generación de patrones de organización durante la interrelación socioemocional madre-hijo, bajo cambios en los estados emocionales de la madre y bajo modificaciones debidas al desarrollo infantil. Los hallazgos de esta primera fase muestran algunas diferencias en las dinámicas socio-emocionales en cada uno de los estados emocionales maternos, al igual que regularidades en la variación por estado emocional. La investigación sugiere ampliar el marco teórico hacia modelos de componentes emocionales y modelos neurobiológicos, y la inclusión de modelos matemáticos de predicción y de análisis de series de tiempos.

\section{Palabras clave}

Desarrollo emocional, sistemas dinámicos, interacción madre-hijo.

\begin{abstract}
Advances in the psychology of emotions and the developmental psychology have demanded further changes, both theoretically and methodologically, to describe and explain the variability of children's responses to changes taking place in the socio-emotional dynamics with the mother. A result of these changes is the integration of dynamic systems to the theoretical descriptions of emotional development in psychobiological and socio-biological assumptions. This study aimed to investigate the possible use of dynamic system models to generate patterns of organization for the social-emotional mother-child relationship, under changes in the mother's emotional states, and under changes due to the child development. The findings of this first phase show some differences in socio-emotional dynamics in each of the maternal emotional states, as well as regularities in the variation per emotional state. The investigation suggests expanding the theoretical framework to models of emotional components and neurobiological models, and the inclusion of mathematical models of forecasting and time series analysis.
\end{abstract}

\section{Keywords}

Emotional Development, Dynamic Systems, Mother-Son Interaction. 


\section{Introducción}

Las teorias sobre las emociones han tenido grandes modificaciones en las últimas décadas. La mayoría de ellas son resultado de los cambios de paradigma en la ciencia psicológica desde los años setenta (Camras \& Witherington, 2005). De un conductismo radical, que dejaba de lado las variables no observables, se pasó a una psicología cognitiva, interesada en los mecanismos internos que podían o no resultar en la respuesta comportamental observada. Lo anterior trajo consigo la idea de programas de control o sistemas internos especificos para la emoción. Estas concepciones estuvieron alimentadas por las investigaciones en ciencia fisiológica, desde donde se identificaban los sitios específicos, corticales o subcorticales, correspondientes a distintos procesos psicológicos, entre ellos los relacionados con las emociones.

Los modelos tradicionales, que veían las emociones como epifenómenos, producto de la cognición y de los estados de activación ("arousal"), fueron reemplazados por las teorías neo-darwinianas, para quienes la emoción tenía una identidad propia y podría nutrirse, a su vez, de modelos basados en la fisiología y en los estudios transculturales como los de Ekman e Izard sobre las expresiones faciales prototipicas (Izard, 1971; Ekman, 1972).

Con el tiempo, los teóricos neo-darwinistas recurrieron a los psicólogos del desarrollo debido a la necesidad de dar significado a las medidas de las emociones en niños pequeños que aún no habían alcanzado la etapa verbal. Izard y sus colegas (2000), describieron un conjunto de expresiones faciales en niños, que podian ser usadas para identificar sus estados emocionales. Estas expresiones tendrian un gran parecido a las expresiones emocionales prototípicas descritas en adultos (Camras \& Witherington, 2005).

Sin embargo, estos hallazgos fueron cuestionados por los teóricos funcionalistas, para quienes las emociones debían ser definidas como procesos destinados al logro, mantenimiento o cambio de las relaciones del organismo con algunos aspectos de su medio. En esta relación se podian o no generar expresiones faciales emocionales prototípicas, como aparece en los procedimientos de violación de expectativa presentados por Camras (2000).
Hallazgos distintos sobre la variabilidad de la respuesta emocional infantil, especialmente en la labilidad ${ }^{2}$ de la conducta expresiva infantil, llevaron a algunos autores a proponer que la explicación de la organización de la respuesta emocional podría ser similar a la requerida por una aproximación de los sistemas dinámicos (De Weerth et al., 1999; Lewis, 2000). Investigaciones como las de Fogel y Thelen (1987) y Fogel (1988), aplicaban la perspectiva de los sistemas dinámicos al estudio de la expresión emocional infantil en el trasfondo de la investigación micro-analitica de la interacción diádica madre-hijo de Kaye y Fogel (1980). Estas investigaciones eran especialmente sensibles a la variabilidad en los detalles finos de la conducta organizada cuya explicación era rechazada por las teorias de las emociones de los modelos neo-darwinianos.

Fogel y Thelen (1987) argumentaban que la variabilidad observada en la morfología, tiempo, contextos evocadores, y función social de esas conductas expresivas tenian implicaciones significativas para la comprensión de la emoción infantil y su desarrollo. Los autores también subrayaban la heterocronicidad en el desarrollo de la respuesta emocional infantil (por ejemplo, la aparición temprana de ciertas sonrisas, separadas de otros componentes que se asociaban tradicionalmente al estado emocional de alegría). La conclusión de estos investigadores señalaba que apelar a un organizador central era inadecuado para explicar esos aspectos importantes de la conducta expresiva infantil.

En resumen, los primeros proponentes de la aproximación de los sistemas dinámicos al estudio de la emoción y del desarrollo emocional intentaron apoyar su posición en cinco puntos:

1. La existencia de fenómenos observados que explicitamente contradecian las teorias neodarwinistas prevalecientes sobre la emoción y el desarrollo emocional.

2. La existencia de fenómenos observados que presumiblemente tenían significancia emocional

$2 \quad$ Entiéndase labilidad emocional como el conjunto de alteraciones en la manifestación de la afectividad (llantos, risas inapropiadas 0 , en general, respuestas emocionales desproporcionadas como reacción a la afectación física). 
pero que no se incluian dentro de las teorias prevalecientes de la emoción.

3. La variabilidad inexplicada en respuestas asociadas con la misma emoción (por ejemplo variabilidad en el llanto y en la risa).

4. La aparente capacidad de los conceptos de los sistemas dinámicos para explicar las observaciones de las cuales las teorías prevalecientes no proveían cuenta satisfactoria (por ejemplo heterocronicidad en el desarrollo de los componentes de la respuesta emocional).

5. La insatisfacción inherente con las teorías que dejaban el control de la conducta en un agente central.

\section{Aproximaciones conceptuales del desarrollo emocional y los sistemas dinámicos}

Hasta el momento, las perspectivas mejor elaboradas que relacionan los sistemas dinámicos con la emoción, han sido la Teoría de proceso social de Fogel (Fogel et al., 1992, 2000), y el modelo de integración de la relación cognición-emoción con una perspectiva neural de la emoción de Lewis (2005a, 2005b). Ambas comparten la característica más importante de los sistemas dinámicos: la auto-organización; sin embargo difieren en ciertas características.

La propuesta de Fogel se puede resumir en tres ejes conceptuales importantes: I. Las emociones son sistemas auto-organizados de componentes que interactúan. II. Las emociones emergen en secuencias de interacción social situadas contextualmente. Y III. El gradiente de información (variaciones en la intensidad de la activación) es una dimensión importante de la emoción en la infancia temprana, que precede el desarrollo de las categorias emocionales discretas. Según el mismo autor, las transiciones emocionales ocurren cuando un patrón emocional estable sufre una ruptura debido a un cambio crítico en uno de sus componentes, o en la adición de un nuevo componente al sistema. Dependiendo de la escala de tiempo involucrada, tales transiciones llevan a la construcción de un nuevo episodio emocional o una nueva fase de desarrollo (Fogel et al., 1992).
Al igual que Fogel, Lewis (2000) considera la emoción como un proceso dinámico auto-organizado con transiciones en diferentes escalas de tiempo pero, a diferencia de aquel, identifica un conjunto de componentes emocionales específicos que residen en el individuo. Además, Lewis integra los modelos neurobiológicos y psicológicos de la emoción para proponer un mapeo estructural entre ellos y una relación funcional aprehensible desde los principios de los sistemas dinámicos (Lewis, 2005a). De esta forma, Lewis se centra más en los aspectos del funcionamiento neuronal involucrado en la generación y regulación de la emoción, mientras que Fogel se focaliza en las transacciones sociales interpersonales que dan origen a los procesos emocionales.

\section{Aplicaciones empíricas de los sistemas dinámicos al estudio del desarrollo emocional}

Las estrategias de investigación de la relación entre desarrollo emocional y sistemas dinámicos han sido resumidas por Thelen y Smith (1994, 2003) en una serie de seis pasos. El primer paso es establecer una variable colectiva de estudio, la cual puede entenderse como una variable observable que refleja el estado del sistema y cuya medida captura las interrelaciones variantes entre un grupo de componentes de bajo orden. El segundo paso en la investigación en sistemas dinámicos implica la identificación de atractores o estados de atracción, los cuales caracterizan una variable colectiva entre diferentes condiciones y diferentes periodos de tiempo.

Los pasos uno y dos son seguidos por el mapeo de la estabilidad temporal y el cambio en la variable colectiva, ya sea a través del análisis microgenético de la variable en tiempo real o a través del estudio longitudinal de la variable en el tiempo del desarrollo (paso tres). Una vez que la trayectoria sea establecida para la variable colectiva, las transiciones de fase o cambios de fase pueden ser identificados en términos de cuando estos ocurren. Las transiciones de fase demarcan cambios de un patrón a otro en el sistema (paso cuatro). El paso cinco involucra la identificación de factores potenciales que podrian subyacer a esas transiciones (llamados parámetros de control). Un último paso implica la manipulación experimental de 
estos parámetros para propósitos de confirmar su estatus (Thelen y Smith, 1998).

Teniendo en cuenta tanto los órdenes teóricos como empíricos presentados anteriormente para el estudio del desarrollo emocional desde la perspectiva de los sistemas dinámicos, se consideró relevante apoyar las teorias psicobiológicas y psicosociales expuestas por Lewis y Fogel, y entrar en el abordaje empírico propuesto por Thelen y Smith para asi describir, desde esta perspectiva, la interrelación madre-hijo bajo cambios emocionales de uno de los componentes de la díada y el efecto sobre el otro componente a lo largo del tiempo.

A partir de estas consideraciones surge la siguiente pregunta de investigación: ¿Sigue la interrelación madre-hijo, bajo cambios en los estados emocionales de la madre y bajo cambios en el desarrollo del niño, patrones de organización posibles de describir en la perspectiva de los sistemas dinámicos?

\section{Método}

\section{Participantes}

Cuatro diadas conformadas por cuatro madres de edades entre los 22 y los 28 años de edad, y cuatro niñas entre los once y los veinte meses. Todas las niñas nacieron a término, sin complicaciones serias, y la madre era la cuidadora primaria. Las madres habian permanecido en el hogar la mayor parte del tiempo durante el primer año de vida. La totalidad de la muestra fue de clase media. Debido a la dificultad de consecución de la muestra, la selección de la misma fue intencional. Las medidas de registro se tomaron con dos meses de diferencia, haciendo que la edad minima en la toma inicial de datos fuese de trece meses y en la final de 24. Se tomó como criterio de exclusión niños menores de diez meses y mayores a 22. Esto para garantizar que la muestra final de niños no superara los dos años de edad.

\section{Instrumentos}

Para el registro de las interacciones diádicas se utilizó una cámara de video HDD marca Panasonic SDR-H80. Para la grabación de las entrevistas a las madres y reproducción de las mismas, se utilizó una grabadora de audio digital marca Sony IDC-PX720 y unos audifonos de auricular.

\section{Procedimiento general}

Siguiendo la propuesta metodológica de investigación por pasos de Thelen y Smith (1994, 1998), y el modelo de análisis del desarrollo emocional de Lewis et al. (1999, 2004), se tomaron dos variables observables de medida como variables colectivas de estudio, estas fueron el ángulo de la mirada del niño y su intensidad emocional.

Para capturar las variables tanto en tiempo real como en tiempo de desarrollo (Smith y Thelen, 2003), se procedió a un registro filmico de la interacción entre la madre y el hijo, máximo un minuto por sesión durante dos sesiones separadas entre sí por dos meses de diferencia. El análisis en tiempo real implicó un análisis microgenético segundo a segundo de la dinámica de interacción sobre las dos variables mencionadas.

Luego de esto se procedió a la graficación de la dinámica diádica, a la identificación de los atractores y a la identificación de posibles estados de fase. Sin embargo no se llegó a los pasos cinco y seis de la propuesta de Thelen y Smith (1994, 1998).

\section{Procedimiento de grabación}

Para la investigación se contó con un total de ocho sesiones de grabación de audio y video, distribuidas en las cuatro diadas, para un total de dos sesiones por diada, separada sesión por sesión con dos meses de diferencia.

Cada sesión tenía un momento previo a la videograbación donde se le pedia a la madre que relatara dos momentos de su vida donde se evocaran estados de alegría y tristeza. Estos relatos debian ser lo más vividos posibles y debían superar, como mínimo, un minuto. La separación entre cada relato debía ser de mínimo cinco minutos. Se tomaron estos dos estados dada la facilidad en su evocación y la fácil evaluación de la expresión emocional según criterios de Izard (1971). 
Luego de esta grabación se invitaba a la díada a la cámara de Gesell. A la madre se le colocaban los audifonos y se le reproducía la grabación de audio, previamente capturada por entrevista, y se procedía a la videograbación. Ésta no debía superar los dos minutos. Los niños se sentaban junto a la madre y la cámara se centraba en los rostros de la madre y el hijo. A las madres se les instruyó para que, durante el proceso de evocación, no intercambiaran palabras con sus hijos.

\section{Procedimiento de codificación y construc- ción de la rejilla}

Siguiendo el procedimiento estipulado por Lewis, Lamey \& Douglas (1999), para cada sesión se codificó el ángulo de la mirada y el nivel del estado emocional en dos escalas ordinales de cinco puntos. Las variables se codificaron de forma separada segundo a segundo, durante las dos sesiones-diada por dos evaluadores ciegos. Los estados emocionales se puntuaron desde un estado neutro a un estado emocional puro (triste o alegre) siguiendo el patrón de registro de expresión facial de Izard (1979). El ángulo de la mirada se puntuó desde la mirada completamente frontal hasta una mirada de evitación completa, representando gradaciones de interés-desinterés, similares a las sugeridas por Beebe y Stern (1977).

Para la puntuación del ángulo de la mirada se tomaron segmentos exactos de un minuto y se fragmentaron en imágenes de un segundo cada una para un total de sesenta imágenes por sesión. Cada segundo de grabación era puntuado por los dobles ciegos en las dos categorias (Rho de Spearman $=0,759, p=$ 0,000 intensidad emocional; Rho de Spearman $=0,760, p=0,000$ ángulo de la mirada).

\section{Procedimiento de Análisis}

Para esta primera fase de la investigación se utilizó un modelo similar al planteado por Lewis et al. $(1999,2004)$ para la representación de las rejillas espacio estado (ver gráfica 1), y se utilizó el software de código libre, "processing" (http://processing.org), para la graficación de los cambios en rejillas que representaban tanto la intensidad emocional como el ángulo de la mirada. Las comparaciones entre momentos y entre estados se hicieron de manera descriptiva apelando a las formas de evolución de las dinámicas de un momento a otro y de un estado emocional a otro.

\section{Gráfica 1. Graficación de LAS REJILLAS espacio-estado}

2 Months
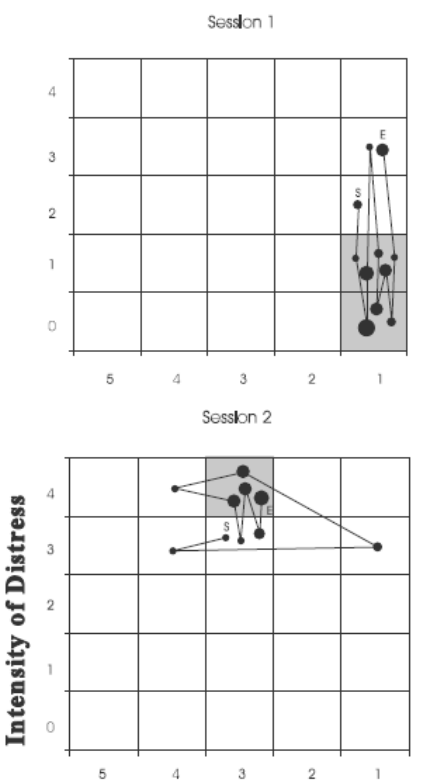

6 Months
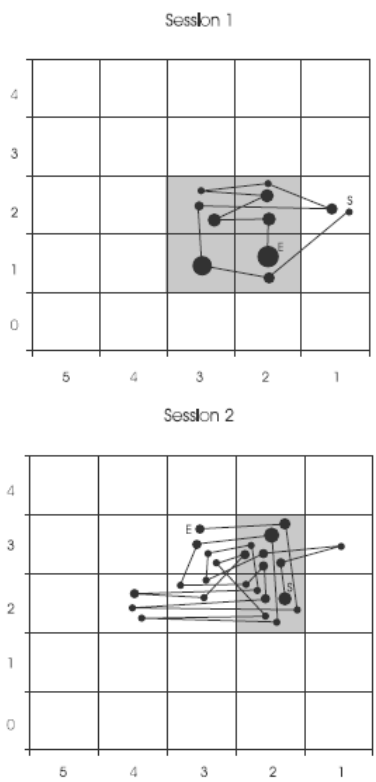

Fuente: Imagen tomada de Lewis, Lamey y Douglas (1999). 


\section{Resultados}

Todos los resultados se interpretaron de manera diferencial tanto por estado emocional (alegre o triste) entre distintos participantes (comparación intersujeto), como a nivel intrasujeto en los distintos momentos de observación.
En la comparación intrasujeto para un mismo estado emocional, se aprecian modificaciones interesantes que demuestran cambios relativos de un mes a otro. Para el caso de la alegría, estas modificaciones implican cambios de estados relativamente estables frente a la evocación emocional de la madre durante el primer momento de observación, a estados relativamente inestables en el segundo momento (ver gráficas 2, 3 y 4).

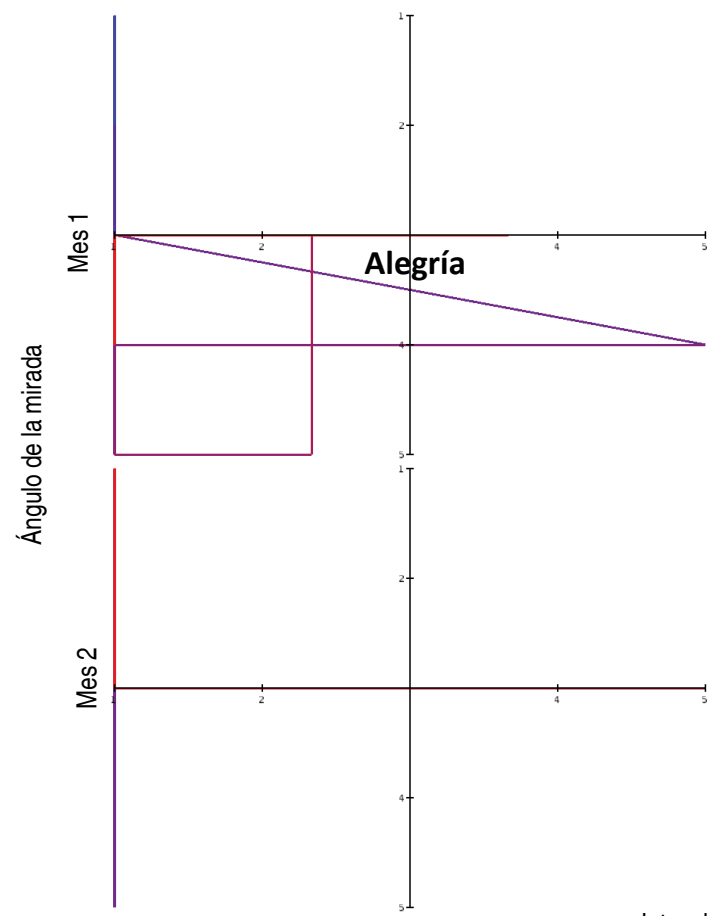

Intensidad emocional

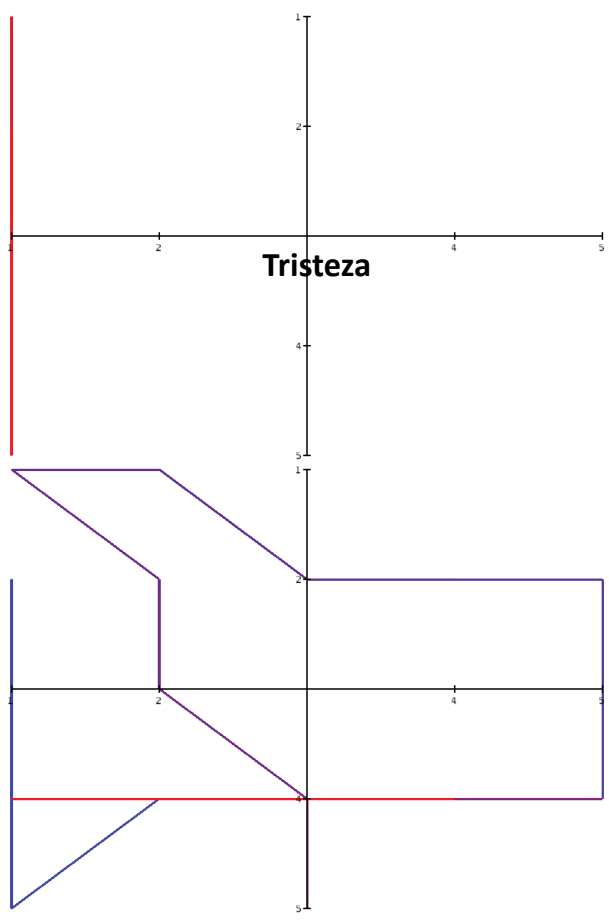


GrÁFICA 3. IMAGEN DE DESEMIPEÑOS DE LA PARTICIPANTE 2
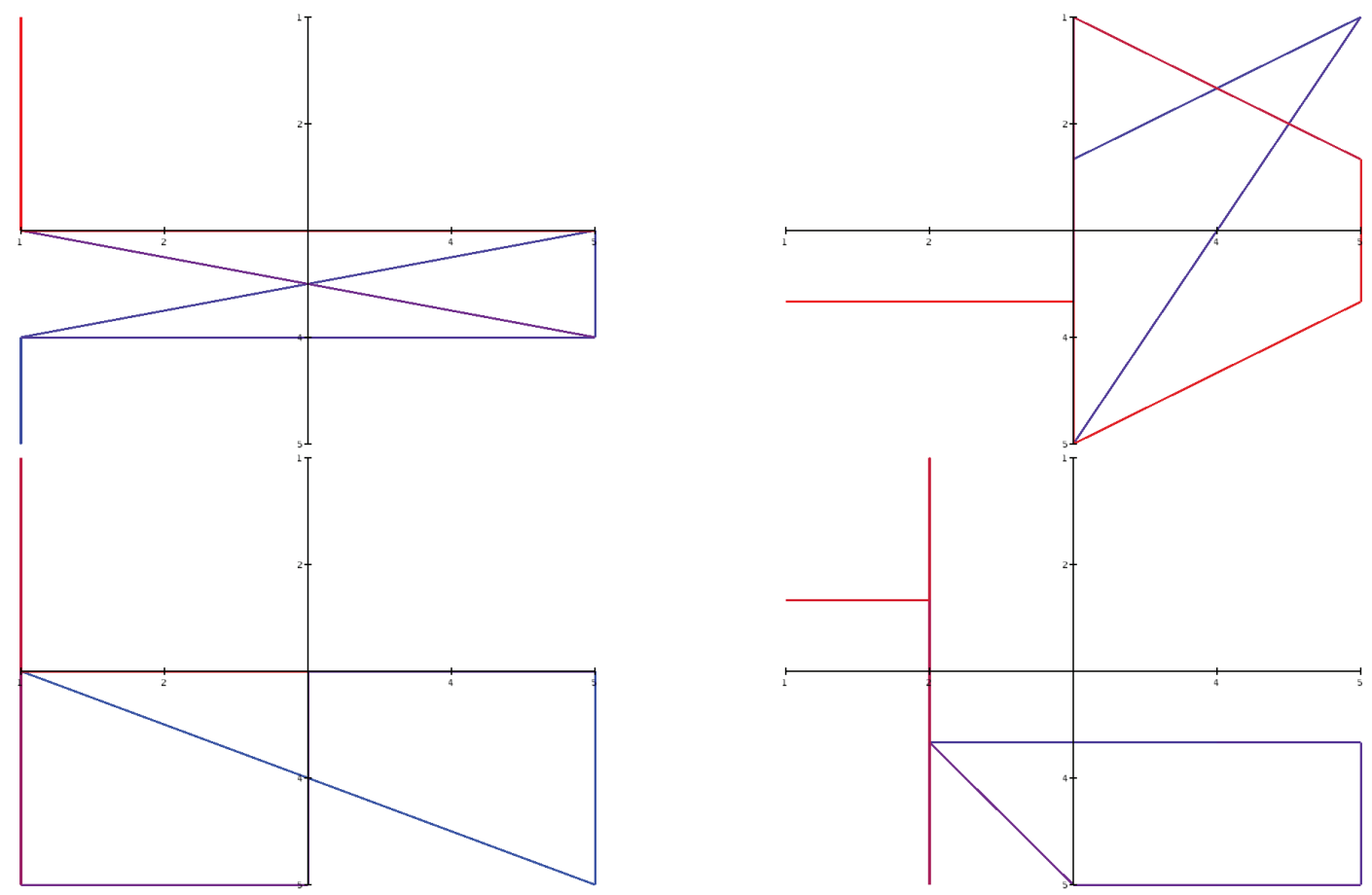

GráFICA 4. IMAGEN DE DESEMPEÑOS DE LA PARTICIPANTE 4.
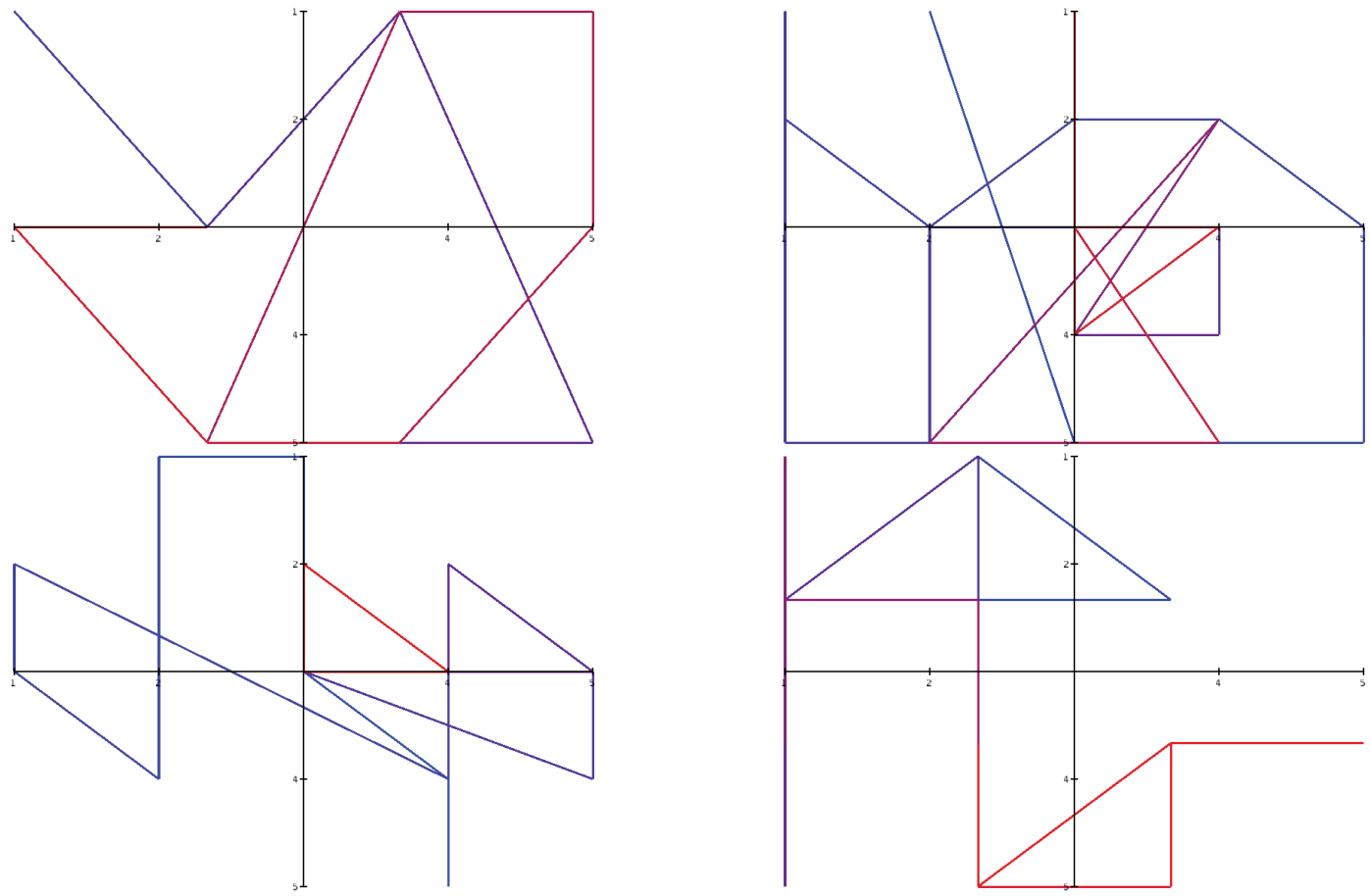
Sin embargo, la comparación entre diadas por edad (intersujeto) para el mismo estado emocional materno de alegria, sugiere saltos cualitativos en estadios tempranos del desarro1lo, de momentos más inestables a momentos más estables.

El estado emocional materno de tristeza tiene un efecto distinto en la dinámica emocional del niño, siendo visibles durante el primer momento de observación dinámicas aleatorias tanto de evitación como de intensidad emocional, con patrones relativamente similares durante el segundo momento (ver gráficas $2,3$ y 4$)$.

\section{Conclusiones}

Los resultados comparativos intrasujetos e intersujetos muestran diferencias en la evolución de la dinámica socioemocional madre-hijo durante el primery segundo mes de observación para los dos estados emocionales estudiados. Los cambios en el estado emocional de alegría de la madre evidencian mayores dinámicas de auto-organización en los niños, mientras que estos cambios durante estados emocionales de tristeza reflejan mayores constancias organizacionales tendientes a la evitación del niño al estado de la madre.

Las modificaciones intrasujeto en el tiempo de desarrollo para el estado emocional de alegría, parecerían señalar un proceso de independencia emocional por efecto del desarrollo como el propuesto por von Salisch (2001), mientras que los cambios de inestabilidad a estabilidad, en momentos distintos para el mismo estado emocional, en díadas más jóvenes, puede interpretarse como fenómenos de regulación del estado emocional propio con el ajeno, en lo que podría llamarse momentos de sincronización emocional (Lewis et al., 1999; De Weerth y van Geert, 2002), que luego irian cambiando en la medida de la relación del niño con el mundo de otros distintos al cuidador (von Salisch, 2001).

Las diferencias emocionales de tristeza intrasujeto, sugieren dinámicas distintas derivadas de las características particulares de los componentes emocionales que intervienen en las relaciones socioemocionales tempranas. Algunas de estas diferencias componenciales ya han sido relatadas en la literatura (Lewis y Granic, 2000; Messinger et al., 2001) y evidencian la necesidad de considerar, en estas dinámicas, aspectos o componentes distintos involucrados en la interacción durante el desarrollo emocional, como las expresiones faciales y las respuestas psicofisiológicas diferenciadas, que pueden ser importantes durante la generación de mecanismos sociales que permitan un desarrollo de competencias emocionales adecuadas.

Si bien el seguimiento de la metodologia de investigación propuesta por Thelen y Smith (1994, 1998), y de los modelos de Lewis $(1999,2004)$ resultan interesantes por cuanto permiten la descripción del desarrollo en términos cercanos a los sistemas dinámicos, aún faltan, para un segundo momento, ampliaciones teóricas y metodológicas que sirvan no sólo a la descripción del cambio durante la interacción socioemocional madre-hijo, sino también a la descripción y comprensión de patrones de cambio o parámetros de control que intervienen en estos procesos de cambio emocional. Una revisión más exhaustiva sugiere tener en cuenta modelos teóricos complementarios como el modelo de patrón componencial presentado por Scherer (Sander, Grandjean \& Scherer, 2005); la inclusión, tanto teórica como metodológica de la propuesta neurobiológica de Lewis (2002, 2005a; Lewis y Stieben, 2004; Lewis y Todd, 2005; y Lewis et al., 2006); el uso del análisis multivariado propuesto por Granic et al. (2002); y el modelo teórico y metodológico para comprender la comunicación emocional propuesto por Fogel et al. (Hsu y Fogel, 2003; Lavelli y Fogel, 2002, 2005). Sólo cuando se logre la consideración e integración de estos elementos podremos tener una teoría más amplia que nos ayude no sólo a describir los patrones de cambio, sino también a comprender los mecanismos que lo generan.

\section{Agradecimientos}

Se agradece la colaboración del matemático Camilo Rey, docente de la Facultad de Ingenieria de la Institución Universitaria Politécnico Grancolombiano, quien ayudó en la graficación de los datos aquí presentados. 


\section{Referencias}

1. Beebe, B. \& Stern, D.N. (1977). Engagement-disengagementand early object experiences. En M. Freedman \& S. Grand (Eds.), Communicative structures and psychic structures (pp.35-55). New York: Plenum.

2. Camras, L. A. (2000). Surprise!: Facial expressions can be coordinative motor structures. En M. Lewis \& I. Granic (Eds.), Emotion, development and selforganization (pp. 100-124). New York: Cambridge University Press.

3. Camras, L. A. \& Witherington, D. C. (2005). Dynamical systems approaches to emotional development. Developmental Review, 25, 328-350.

4. De Weerth, C. \& van Geert, P. (2002). Changing patterns of infant behavior and mother-infant interaction: Intra- and interindividual variability. Infant Behavior \& Development, número 24, 347-371.

5. De Weerth, C., van Geert, P. \& Hoijtink, H. (1999). Intraindividual variability in infant behavior. Developmental Psychology, número 35(4), 1102-1112.

6. Ekman, P. (1972). Universals and cultural differences in facial expressions of emotion. En J. Cole (Ed.), Nebraska Symposium on Motivation: Vol. 19. Current Theory and Research in Motivation (pp. 207-283). Lincoln: University of Nebraska Press.

7. Fogel, A. (1988). Cyclicity and stability in mother-infant face-to-face interaction: A comment on Cohn and Tronick (1988). Developmental Psychology, 24(3), 393-395.

8. Fogel, A. \& Thelen, E. (1987). Development of early expressive and communicative action: Reinterpreting the evidence from a dynamic systems perspective. Developmental Psychology, 23(6), 747-761.
9. Fogel, A., Nelson-Goens, C., Hsu, H.-C. \& Shapiro, A. F. (2000). Do different infant smiles reflect different positive emotions? Social Development, 9(4), 497-520.

10. Fogel, A., Nwokah, E., Dedo, J., Messinger, D., Dickson, K., Matusov, E., y cols. (1992). Social process theory ofemotion: A dynamic systems approach. Social Development, 1(2), 122-142.

11. Granic, I. \& Lamey, A. V. (2002). Combining dynamic systems and multivariate analyses to compare the mother-child interactions of externalizing subtypes. Journal of Abnormal Child Psychology, 30(3), 265-283.

12. Hsu, H. \& Fogel, A. (2003). Stability and transition in mother-infant face-toface communication during the first 6 months: A microhistorical approach. Developmental Psychology, 39, (6), 1061-1082.

13. Izard, C. E. (1971). The face of emotion. New York: Appleton-Century-Crofts.

14. Izard, C. E., Ackerman, B. P., Schoff, K. M. \& Fine, S. E. (2000). Self-organization of discrete emotions, emotion patterns, and emotion-cognition relations. En M. D. Lewis \& P. Granic (Eds.), Emotion, development, and selforganization: Dynamic systems approaches to emotional development (pp. 15-36). Cambridge, UK: Cambridge University Press.

15. Izard, C.E. (1979). The maximally discriminative facial move-ment coding system (Max). Newark, NJ: InstructionalResources Center, University of Delaware.

16. Kaye, K., \& Fogel, A. (1980). The temporal structure of face-to-face communication between mothers and infants. Developmental Psychology, 16, 454-464. 
17. Lavelli, M., \& Fogel, A. (2002). Developmental changes in mother-infant face-to-face communication: Birth to 3 months. Developmental Psychology, 38(2), 288-305.

18. Lavelli, M., \& Fogel, A. (2005). Developmental changes in the relationship between the infant's attention and emotion during early face-to-face communication: The 2-month transition. Developmental Psychology, 41(1), 265-280.

19. Lewis, M. D. (2000). The promise of dynamic systems approaches for an integrated account of human development. Child Development, 71 (1), 36-43.

20. Lewis, M. D. (2002). The dialogical brain: Contributions of emotional neurobiology to understanding the dialogical self. Theory \& Psychology, 12(2), 175-190.

21. Lewis, M. D. (2005a). Bridging emotion theory and neurobiology through dynamic systems modeling. Behavioral and Brain Sciences, 28, 169-245.

22. Lewis, M. D. (2005b). Self-organizing individual differences in brain development. Developmental Review, 25, 252-277.

23. Lewis, M. D. \& Granic, P. (2000). Emotion, development, and selforganization: Dynamic systems approaches to emotional development. Cambridge, UK: Cambridge University Press.

24. Lewis, M. D. \& Stieben, J. (2004). Emotion regulation in the brain: Conceptual issues and directions for developmental research. Child Development, 75(2), 371-376.

25. Lewis, M. D., \& Todd, R. M. (2005). Getting emotional: A neural perspective on emotion, intention, and consciousness. Journal of Consciousness Studies, número 12, 210-235.
26. Lewis, M. D., Lamey, A., \& Douglas, L. (1999). A new dynamic systems method for the analysis of early socioemotional development. Developmental Science, 2 (4), 457-475.

27. Lewis, M. D., Lamm, C., Segalowitz, S. J., Stieben, J., \& Zelazo, P. D. (2006). Neurophysiological correlates of emotion regulation in children and adolescents. Journal of Cognitive Neuroscience, 18(3), 430-443.

28. Lewis, M. D., Zimmerman, S., Hollenstein, T. \& Lamey, A. V. (2004). Reorganization in coping behavior at 1 $1 / 2$ years: Dynamic systems and normative change. Developmental Science, 7(1), 56-73.

29. Messinger, D. S., Fogel, A. \& Dickson, K. L. (2001). All smiles are positive, but some smiles are more positive than others. Developmental Psychology, 37(5), 642-653.

30. Sander, D., Grandjean, D., \& Scherer, K. (2005). A systems approach to appraisal mechanisms in emotion. Neural Networks, número 18, 317-352

31. Smith, L. \& Thelen, S. (2003). Development as a dynamic system. TRENDS in Cognitive Sciences, 7 (8), 343-348.

32. Thelen, E. \& Smith, L. B. (1994). A dynamic systems approach to the development of cognition and action. Cambridge, MA: MIT Press.

33. Thelen, E. \& Smith, L. B. (1998). Dynamic systems theories. En W. Damon \& R. M. Lerner (Eds.). Handbook of child psychology: Vol. 1. Theoretical models of human development (pp. 563-634). New York: Wiley.

34. Von Salisch, M. (2001). Children's emotional development: Challenges in their relationships to parents, peers, and friends. International Journal of Behavioral Development, 25 (4), 310-319. 